\title{
A Motion- and Sound-Activated, 3D-Printed, Chalcogenide-Based Triboelectric Nanogenerator
}

\author{
Mehmet Kanik, Mehmet Girayhan Say, Bihter Daglar, Ahmet Faruk Yavuz, \\ Muhammet Halit Dolas, Mostafa M. El-Ashry, and Mehmet Bayindir*
}

Scavenging waste energy is an alternative prominent solution, which may play an important role regarding the world energy problems. Nowadays, many portable electronic devices are produced and they require very low power for functioning. ${ }^{[1,2]} \mathrm{A}$ compact mobile device design is assumed to be light weight with a long-lasting battery. ${ }^{[3,4]}$ However, the end of the battery life is inevitable. Besides, a mobile device should be plugged into an energy-distribution source in order to charge its battery, which limits the mobility of the user. A portable energyharvesting system is a considerable energy source that never depletes. There are many piezoelectric nanogenerators proposed for this purpose. ${ }^{[5-9]}$ Drawbacks of piezoelectric materials are the requirements of complex and high-cost manufacturing processes such as electric polarization using high electric field strengths to obtain high-efficiency piezoelectric materials, high brittleness, and toxic elemental composition. ${ }^{[10]}$ In order to overcome these drawbacks, very sophisticated processing conditions and techniques are required. ${ }^{[5,11]}$

In the last few years, many researchers have reported that nanogenerators based on the triboelectric effect are promising energy sources; they can harvest mechanical energy from human motions, sound, hydraulic pressure, and wind using the nanostructured surfaces of the dielectric materials. ${ }^{[12-21]}$ The triboelectric effect has been known for a couple of centuries, but it has not been clearly understood yet. However, there are a few significant attempts based on charged atom transfer, ${ }^{[22,23]}$ ion transfer, ${ }^{[24]}$ and the bonding of radicals ${ }^{[25,26]}$ to explain the mystery behind the triboelectric effect. When micro- and nanostructured dielectric surfaces are in contact with each other or

M. Kanik, M. G. Say, B. Daglar, A. F. Yavuz,

M. H. Dolas, Dr. M. M. El-Ashry, Prof. M. Bayindir

UNAM-National Nanotechnology Research Center

Bilkent University

06800 Ankara, Turkey

E-mail: bayindir@nano.org.tr

M. Kanik, M. G. Say, B. Daglar, A. F. Yavuz, Prof. M. Bayindir

Institute of Materials Science and Nanotechnology

Bilkent University

06800 Ankara, Turkey

M. H. Dolas, Prof. M. Bayindir

Department of Physics

Bilkent University

06800 Ankara, Turkey

Dr. M. M. El-Ashry

Egypt Nanotechnology Center

Cairo University

12588 Cairo, Egypt

DOI: $10.1002 / \mathrm{adma.201405944}$ separated, there are positive and negative charges generated on coated electrodes depending on the triboelectric polarity of those dielectric materials. Therefore, a perfect design for a triboelectric nanogenerator (TENG) can be achieved by choosing the most distinct materials in terms of triboelectric polarity as well as diminishing of the feature sizes of those materials down to nanometer scale for obtaining maximum contact area. ${ }^{[20]} \mathrm{A}$ list of the triboelectric materials in accordance with their polarity is presented in Table S1, Supporting Information. Working mechanisms of TENG devices are based on two mechanical motion modes, contact and sliding mode. ${ }^{[20]}$ Recent studies reveal that the sliding mode has a better voltage output. ${ }^{[13]}$ However, it requires a more complicated device design. The output voltage of TENGs is enhanced above $1 \mathrm{kV}$ by using different material combinations and device geometries. ${ }^{[12]}$

In this study, we constructed core-shell nanostructures (polyethersulfone (PES) is in the core and $\mathrm{As}_{2} \mathrm{Se}_{3}$ is in the shell) for building a 3D TENG device (Figure 1a-6,a-7). Aluminum tape was used as a substrate and contact electrode for both polyetherimide (PEI) nanopillars and $\mathrm{As}_{2} \mathrm{Se}_{3}$ core-shell nanostructures (Figure 1a-1,a-5). Detailed information about the fabrication of the $\mathrm{As}_{2} \mathrm{Se}_{3}$ core-shell nanostructures and the PEI nanopillars is given in the Supporting Information (see Figure S1). The fluorination process used in the fabrication of the PEI nanopillars and surface modification of the $\mathrm{As}_{2} \mathrm{Se}_{3}$ nanostructures is given in Figure S2 in the Supporting Information. Figure S3 in the Supporting Information illustrates the fabrication process of the nanopillars.

Our device can be stimulated by both motion and acoustic waves at different frequencies. We combined fluorinated $\mathrm{As}_{2} \mathrm{Se}_{3}$ kilometer-long core-shell nanostructures, which are produced using an iterative fiber-drawing technique, with PEI nanopillars, which are produced by a template-based method for the construction of the contact mode TENG in a multilayer fashion. Our device has an output of maximum $1.23 \mathrm{~mW}$ direct current (DC) and $0.51 \mathrm{~W}$ peak power and can power parallel connected 38 LEDs simultaneously. Our chalcogenide-based TENG has maximum $396 \mathrm{~V}$ and $1.6 \mathrm{~mA}$ peak-to-peak output voltage and current, respectively. In addition, a finite element model is developed to explain contact electrification between core-shell nanostructures and nanopillars using COMSOL Multiphysics. A perfect match between analytically calculated open-circuit voltage (OCV) and OCV measurement for a single layer generator was presented.

To enhance the performance of triboelectric devices, there are two important parameters that play a major role in the selection of material combinations: the surface properties and triboelectric polarity. ${ }^{[20]}$ Since the surface properties can be modified using various techniques, the triboelectric polarity is 
a
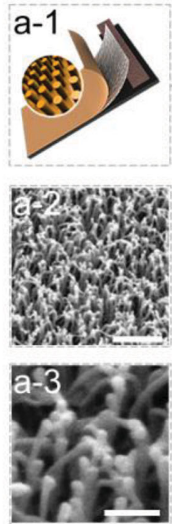

a-4
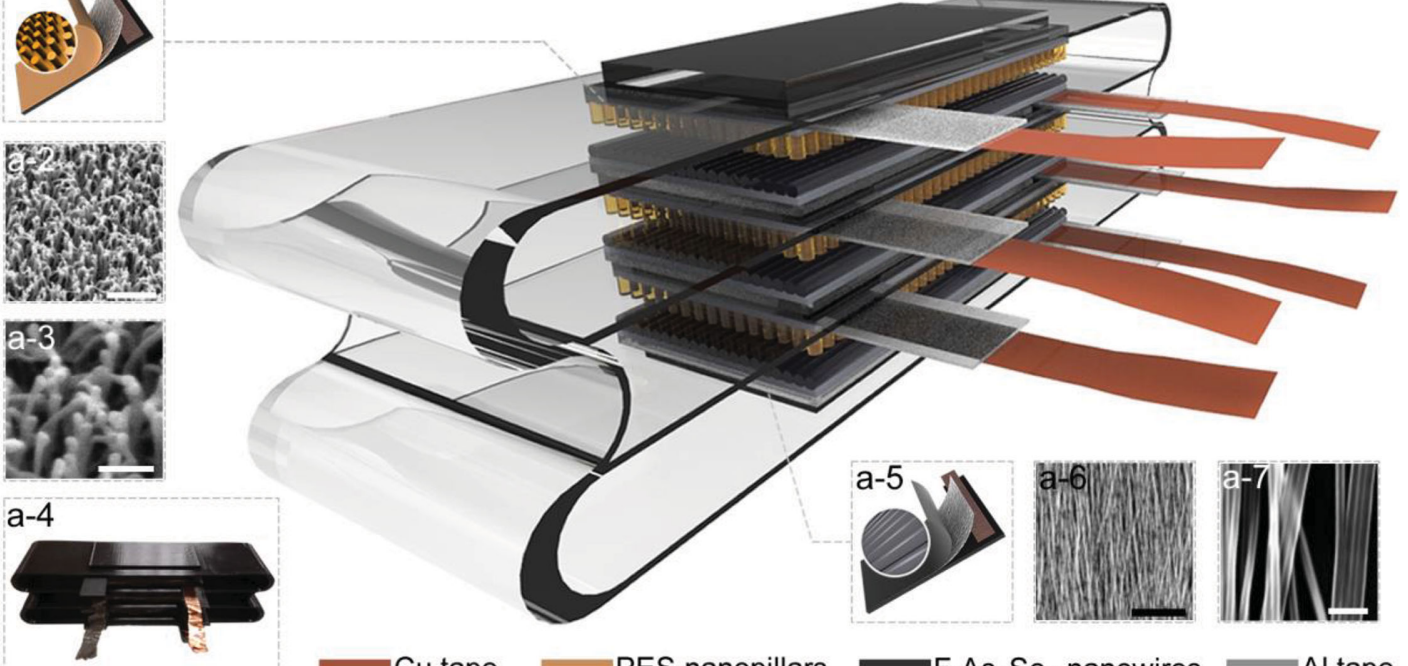

b

\section{Full Seperation}
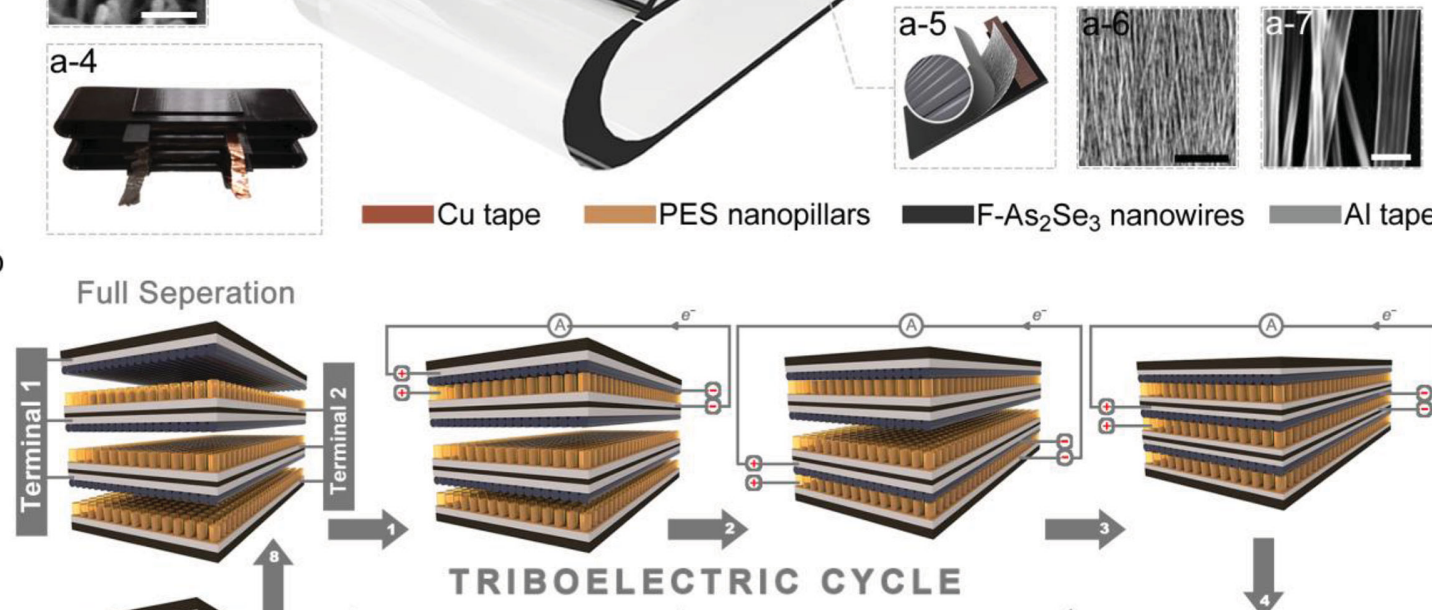

Cu tape

$\mathrm{F}-\mathrm{As}_{2} \mathrm{Se}_{3}$ nanowires

Al tape
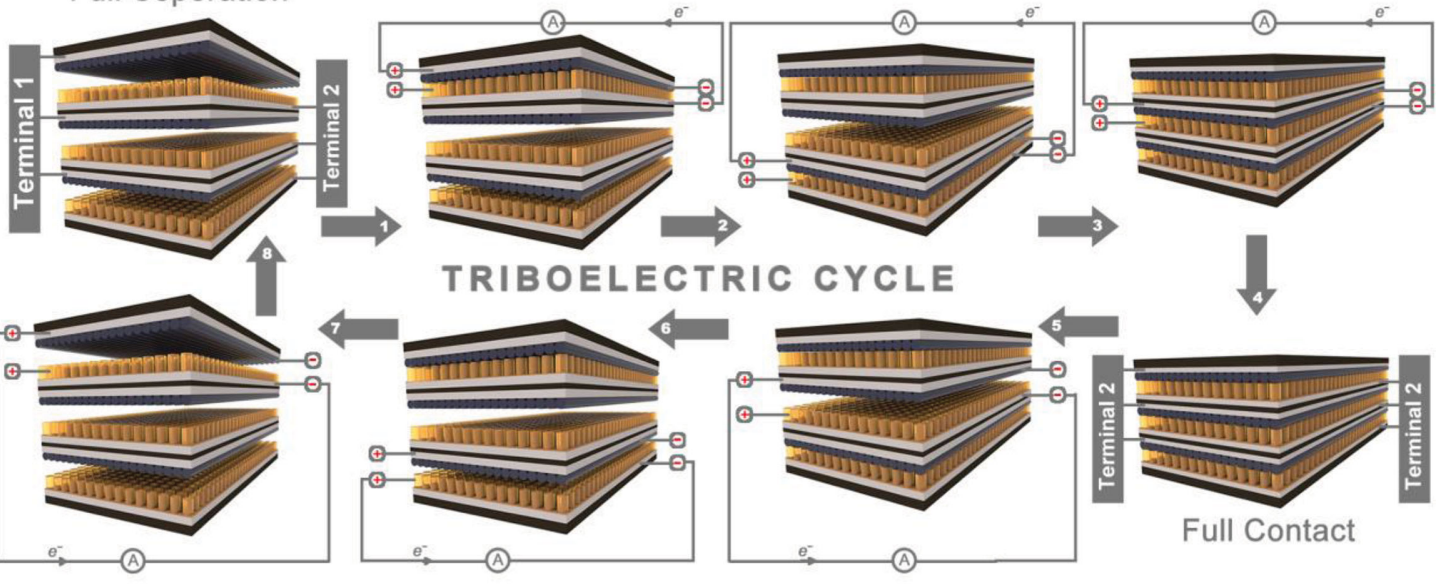

PLA

Al tape

PEI nanopillars

$\mathrm{F}-\mathrm{As}_{2} \mathrm{Se}_{3}$ nanowires

Figure 1. Detailed images of chalcogenide-based 3D-printed MULTENG energy harvesting device and its layers. a) An illustration of a 3D-printed MULTENG energy harvesting device which consists of six alternating polymer and chalcogenide layers. a-1) Representation of layers which consist of PEI nanopillars. a-2) SEM image of PEI nanopillars. Scale bar is $1 \mu \mathrm{m}$. a-3) Higher magnification SEM image of PES nanopillars. Scale bar is $250 \mathrm{~nm}$. a-4) Photograph of the MULTENG device fabricated with the 3D-printing method. a-5) Representation of layers, which consist of $\mathrm{F}-\mathrm{As}_{2} \mathrm{Se}_{3}$ core-shell nanostructure. a-6) SEM image of $\mathrm{F}-\mathrm{As}_{2} \mathrm{Se}_{3}$ core-shell nanostructures. Scale bar is $25 \mu \mathrm{m}$. a-7) Higher magnification SEM image of $\mathrm{F}-\mathrm{As}_{2} \mathrm{Se}_{3}$ core-shell nanostructures. Scale bar is $500 \mathrm{~nm}$. b) Working mechanism of the MULTENG that utilizes the contact mode triboelectrification with external electronic flow in short circuit condition. 1. Motion of the MULTENG from original state to charge generation state. Sharing charges and positive peak current generation (current flow) in unit 1 of the MULTENG. 2. Sharing charges and positive peak current generation (current flow) in unit 3 of the MULTENG. 3. Sharing charges and positive peak current generation (current flow) in unit 2 of the MULTENG. 4. Full contact position. 5. Separation of layers and sharing charges and positive peak current generation (current flow) in unit 2 of the MULTENG. 6. Separation of layers and sharing charges and positive peak current generation (current flow) in unit 3 of the MULTENG. 7. Separation of layers and sharing charges and positive peak current generation (current flow) in unit 1 of the MULTENG. 8. Full separation position.

the most important inherent parameter that need to be considered. We analyzed the triboelectric response of many materials by measuring the OCVs and short circuit currents (SCCs) from $1 \mathrm{~cm} \times 2 \mathrm{~cm}$ samples. The measurement setup and samples are illustrated in Figure S4a-c in the Supporting Information. Measurements were conducted under same conditions (3 g load and at $20 \mathrm{~Hz}$ tapping frequency) for all samples. Since the triboelectric effect is not well known and the triboelectric series is not perfectly and fully characterized, we combined different material sets which are promising for energy harvesting applications. The following materials were combined: carbon-loaded polyethylene (CPE), polycarbonate (PC), polyester (PEST), PEI, polydimethylsiloxane (PDMS), PES, poly(vinylidene flouride) PVDF, fluorinated polyetherimide (F-PEI), fluorinated arsenic selenide $\left(\mathrm{F}-\mathrm{As}_{2} \mathrm{Se}_{3}\right)$, and polytetrafluoroethylene (Teflon) (Table 1). Charge values and details of the measurement protocol are given in the Supporting Information. Teflon presents superior properties in terms of triboelectric properties. 
Table 1. List of dielectric materials in accordance with their triboelectric response as we tested in pairs.

\begin{tabular}{cc}
\hline \multicolumn{3}{|c}{ Triboelectric Polarity } \\
\hline Carbon Loaded Polyethene (CPE) \\
Polycarbonate (PC) \\
Polyester (PEST) \\
Polyetherimide (PEI) \\
Polydimethylsiloxane (PDMS) \\
Polyether Sulphone (PES) \\
Polyvinlylidene Fluoride (PVDF) \\
Flourinated Polyetherimide (F-PEI) \\
Flourinated Arsenic-Selenide (F-As $\mathrm{Se}_{3}$ ) \\
Polytetrafluoroethylene (TEFLON) \\
\hline
\end{tabular}

Unfortunately, it is not compatible with the iterative fiberdrawing process, which is an exciting fabrication technique ${ }^{[11]}$ that enables kilometer-long, parallel oriented, and polymer encapsulated nanostructures to be produced from a variety of materials, including $\mathrm{As}_{2} \mathrm{Se}_{3}{ }^{[27]}$ and some fluorine-rich polymers such as PVDF. ${ }^{[5]}$ In the fabrication, there are at least two materials with compatible properties, a guard polymer jacket and a core material. Materials used in the iterative fiber-drawing process represent unique properties, e.g., low thermal expansion, low melting point (for core material), low glass-transition temperature, and no crystallinity. In this case, the core material is crystalline and the glass-transition temperature of the covering polymer preform should be higher than the melting point of the core material so that the core material can melt in the preform and the liquefied material takes the shape of the fiber (nanowire) during the process. ${ }^{[5,11]}$ Unfortunately, Teflon is a highly crystalline polymer with a high melting temperature, which makes it incompatible with the iterative fiber-drawing process. Unlike Teflon, $\mathrm{As}_{2} \mathrm{Se}_{3}$ is perfectly compatible with the iterative fiber-drawing process because of its low glasstransition temperature, easy processibility, and amorphous structure. ${ }^{[27]}$ In addition, although arsenic is a toxic element, $\mathrm{As}_{2} \mathrm{Se}_{3}$ is an inert intermetallic material with no toxicity, that does not reflect any of the hazardous characteristics of arsenic and has been used in open cancer surgeries in fiber shape. ${ }^{28,29]}$ Although, the voltage peaks obtained from bare $\mathrm{As}_{2} \mathrm{Se}_{3}$ coreshell nanostructures were insignificant, the performance of the core-shell nanostructures was enhanced close to that of Teflon's after core-shell nanostructures were coated with fluorine monolayer. As a result of our experiments, we concluded that the materials with higher fluorine content tend to generate more triboelectric charges.

The triboelectric effect is a surface contact and separation phenomenon, and increasing the surface area also increases the device performance. In addition to an increase in the total surface area by using nanoscale materials, using multilayers is also important to multiply the total converted energy. The working principle of a MULTENG device which consists of six alternating layers is illustrated in Figure 1b. We investigated the synchronism of the three units of MULTENG and voltage response at different frequencies (Figure S5, Supporting Information). To explain the working mechanism, we recorded video of a spare MULTENG during operation (tapping) using a high frame rate (HFR) camera (Video 1, Supporting Information). In addition, we designed a finite element simulation to confirm the HFR video results (Video 2, Supporting Information). Before examination of the working mechanism of the MULTENG, we should recall that the MULTENG has three units which consist of six layers making contact and separation in pairs (unit 1 consists of layer 1 and 2, unit 2 consists of layer 3 and 4, unit 3 consists of layer 5 and 6 ). The force for making contact between the layers of the MULTENG was applied through the top and the MULTENG was fixed from the bottom. The MULTENG started to squeeze when a triggering force was applied. In the early stage of its motion, the distances between the layers started to decrease in units 1 and 3 and slightly increase in unit 2. This is because the shape of the side connections of the three units was designed differently. The curvatures in convex connections of units 1 and 3 contracted and the curvature in concave connection of unit 2 expanded by applied force. Therefore, layers of units 1 and 3 approach each other whereas layers of unit 2 move apart from each other. According to simulation and HFR video recordings, the motions of the three units were at different speeds and this was observed as unit 1 to make contact first while unit 3 was slightly apart and unit 2 was completely separated. Following unit 1, layers of unit 3 were brought in contact position by further pressing on top of the MULTENG. In the last stage, after layers of units 1 and 3 made contact, they transmit force to unit 2 and thus, layers of unit 2 came in contact position. When the applied force was released, the layers of units were separated vice versa with making the contact sequence. First, the layers of unit 2 separated, second, the layers of unit 3 separated, and finally, the layers of unit 1 separated. Considering the fact that a MULTENG should be durable against high cyclic motions and each layer should perform perfect contact and separation, we designed a MULTENG with $175 \mathrm{~mm}$ in length, $50 \mathrm{~mm}$ in width, and $49 \mathrm{~mm}$ in thickness as shown in Figure 1a. A detailed technical drawing of our device is given in Figure S6a-f, Supporting Information. Poly(lactic acid) (PLA) was used as 3D-printing material because it has low density, high flexibility, and low melting temperature. As shown in Figure S6a,d, Supporting Information, there are four $1 \mathrm{~mm}$-thick PLA layers connected to each other with three half circular loops, which provide a good restoring mechanism and flexibility. There are thicker rectangular $(75 \mathrm{~mm}$ in length and $35 \mathrm{~mm}$ in width) rigid regions in the middle of these layers which facilitate making a better contact between layers during the triboelectric cycle. In addition to the main body, six PLA thick layers ( $80 \mathrm{~mm}$ in length and $40 \mathrm{~mm}$ in width) were printed (Figure S6e, Supporting Information) as a holder for the $\mathrm{Al}$ electrodes, $\mathrm{As}_{2} \mathrm{Se}_{3}$ core-shell nanostructures, and PEI nanopillars. $27 \mathrm{~mm}$ long and $500 \mu \mathrm{m}$ thick tails were extended on PLA holders which render the $\mathrm{Cu}$ tape contacts as durable under high cyclic motions. In the assembling process, $\mathrm{Cu}$ tapes (10 $\mathrm{mm}$ in width, $50 \mu \mathrm{m}$ in thickness) were attached on PLA holder surface along the width of holders and extended through 
flexible tails, $50 \mu \mathrm{m}$ thick double-sided Al conductive tapes (40 $\mathrm{mm} \times 80 \mathrm{~mm}$ ) were located on holders in contact with $\mathrm{Cu}$ tapes in all six holders. Three of the holders with $\mathrm{Al}$ electrodes were covered with PEI nanopillars (100 $\mathrm{nm}$ in diameter) (Figure S7a, Supporting Information). $\mathrm{F}-\mathrm{As}_{2} \mathrm{Se}_{3}$ core-shell nanostructures (180 $\mathrm{nm}$ in diameter) were dispersed on the last three of the holders with Al electrodes (Figure S7b, Supporting Information). Finally, PLA holders with nanostructures on top of them were attached to the main body. To our knowledge, this is the first study showing that a chalcogenide material was used for constructing a triboelectric device.

In the fabrication process of nanopillar-decorated PEI films, the melt-infiltration method ${ }^{[30,31]}$ was used (Figure S3, Supporting Information). Here, dissolved PEI behaves as an infiltration phase and the anodized aluminum oxide (AAO) membrane behaves as a template under the base of melt-infiltration. The AAO template was coated with $1 \%(\mathrm{v} / \mathrm{v}) 1 \mathrm{H}, 1 \mathrm{H}, 2 \mathrm{H}, 2 \mathrm{H}$ perfluorodecyl-trichlorosilane (FDTS) in $n$-heptane solution prior to use. ${ }^{[30]}$ Fluorination prevents polymer adherence into the membrane pores. In this process, the AAO membrane, which was grown on the aluminum sheet, was directly used as supported on its aluminum substrate. The template has $5 \mathrm{~cm}$ $\times 10 \mathrm{~cm}$ dimensions with a $100 \mathrm{~nm}$ pore diameter and a $2 \mu \mathrm{m}$ pore depth. PEI was dissolved in dichloromethane (DCM) at a concentration of $0.3 \%(\mathrm{w} / \mathrm{v})$. PEI solution was drop cast on the AAO template and left for solvent evaporation. After the evaporation step, PEI film was peeled off from the surface. Therefore, $4 \mathrm{~cm} \times 8 \mathrm{~cm}$ PEI nanopillar films were obtained for the fabrication of MULTENG.

$\mathrm{F}-\mathrm{As}_{2} \mathrm{Se}_{3}$ core-shell nanostructures were produced using iterative thermal size reduction technique based on fiber drawing (Figure S1, Supporting Information). Initially, we produced a $\mathrm{As}_{2} \mathrm{Se}_{3}$ tube that was $10 \mathrm{~cm}$ in length and $10 \mathrm{~mm}$ in diameter using the sealed ampule method (Figure S8, Supporting Information). In this method, elements were inserted in a quartz tube and vacuum sealed at elevated temperatures to remove all impurities and oxygen as well as the moisture. The mixture in the tube was melted in a rocking oven at $750{ }^{\circ} \mathrm{C}$. After overnight rocking of the samples, the molten material was quenched in tap water for increasing the glass formation by overcooling the material. Before quenching, molten $\mathrm{As}_{2} \mathrm{Se}_{3}$ was centrifuged for the formation of a $10 \mathrm{~cm}$ long hollow core structure with $10 \mathrm{~mm}$ outer diameter and $6 \mathrm{~mm}$ inner diameter. To absorb the impact during the tapping on the MULTENG, we filled inside of the tube with a PES rod which was mechanically extracted out from a PES preform. After three steps of iterative fiber drawing, we obtained $100 \mathrm{~nm}-1 \mu \mathrm{m}$ thick core-shell nanostructures. Detailed SEM images of the nanowires are shown in Figure S9 in the Supporting Information. Finally, nanostructures were coated with self-assembled fluorine monolayer. The fluorination process was repeated as performed in the template coating step. Then, the nanostructures were washed with $n$-heptane and dried at $100{ }^{\circ} \mathrm{C}$ in a vacuum oven.

The MULTENG can be operated using a single hand as shown in Video 3 in the Supporting Information. The performance of the MULTENG was characterized under different conditions. OCV and SCC of the device were recorded using various activation sources such as mechanical tapping, car vibration, and sound. The peak power, power density, and device efficiency were calculated. In addition, the DC power was also calculated, which represents the constant power supply capacity of the MULTENG as a function of the triboelectric device performance and the triboelectric cycle frequency. The OCV and SCC of MULTENG were measured under constant force and tapping frequencies at 1, 2.5, 5, and 7.5 Hz using a custom-made tapping machine with a measurement setup as shown in Figure S10a-c, Supporting Information. The tapping machine consists of a $10 \mathrm{~N} \mathrm{~m}$ motor with a maximum speed of $1360 \mathrm{rpm}$. The base part of the device is stable and the height of the base is manually adjustable. The top part is movable and its frequency and applied load can be adjusted. The power of the motor was calculated using the following equation:

$$
P_{\mathrm{w}}=6.28 \mathrm{Tf} \eta
$$

where $P_{\mathrm{w}}$ is the output power (W), $T$ is the torque $(\mathrm{N} \mathrm{m}), f$ is the frequency $(\mathrm{Hz})$, and $\eta$ is the efficiency. As seen in Figure 2a, the OCV increases with increasing frequency. Variations in the OCV with respect to frequency were basically because of that input power which triggers the MULTENG increase with increasing frequency in our system (Figure S11, Supporting Information). However, the same increasing trend with respect to increasing tapping frequency was not conserved for the SCC values in Figure $2 \mathrm{~b}$. The maximum peak-to-peak OCV was measured as $396 \mathrm{~V}$ at $7.5 \mathrm{~Hz}$ and the maximum peak-to-peak SCC was measured as $1.62 \mathrm{~mA}$ at $2.5 \mathrm{~Hz}$. As seen in Figure 1a and Figure S6 in the Supporting Information, layers of the MULTENG are connected with half circular loops and each layer is long enough to preserve mechanical flexibility. The design resembles a spring in appearance. Actually, the MULTENG base structure functions as a spring to separate layers after contact. The following actions sequentially took place during the measurement: a force was applied from the top of the MULTENG as tapping while the MULTENG was fixed from the bottom, layers were bent until they came in the full contact position, and the force was released and the layers regained the initial position. There was a certain time required for regaining the position. Maximum efficiency was obtained when the tapping frequency was close to or same as the resonance of the MULTENG. In the case of a tapping frequency higher than the resonance frequency of the MULTENG, which means the time delay between two sequential tapping was less than the required time for regaining the initial position of layers, there was no full separation between layers. This causes decrease and fluctuation at higher frequencies. The triboelectric effect is not well understood, but some parts of the effect are perfectly proven in recent studies. ${ }^{[23,25]}$ Basically, when two dielectrics with different triboelectric polarities are brought together, the negatively charged surface becomes more negative, the positively charged surface becomes more positive, and both negative and positive charges can be localized on the surface simultaneously and inhomogeneously. Discharging of these charges after separation of the surfaces is not in the same rate and homogeneity. This phenomenon was perfectly proven using state-of-the-art techniques including the Kelvin force microscopy surface charge scanning technique. ${ }^{[23,25]}$ The same phenomenon naturally occurs in our MULTENG layers and this causes fluctuations. $\mathrm{As}_{2} \mathrm{Se}_{3}$ layers were randomly dispersed on the $\mathrm{Al}$ tape electrode, which 


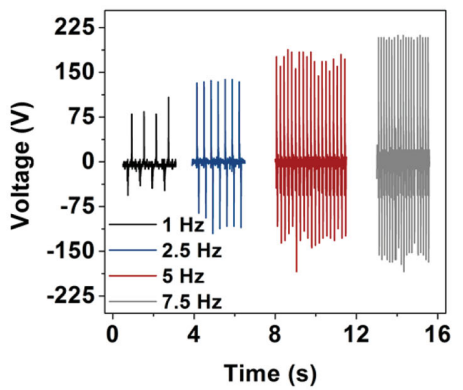

d

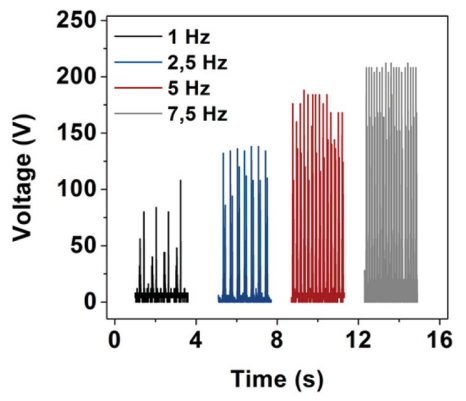

g

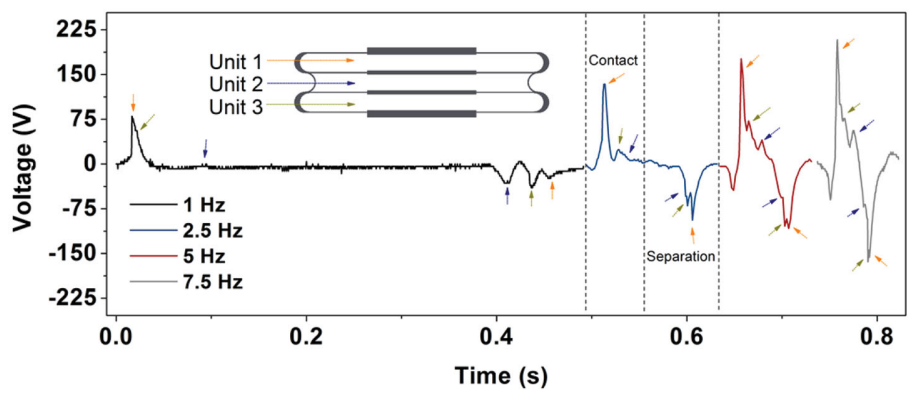

i

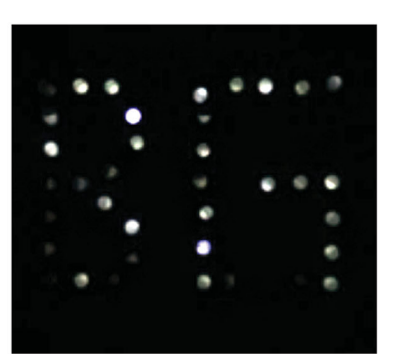

b

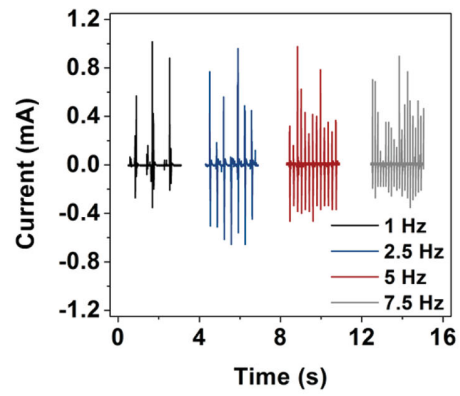

C

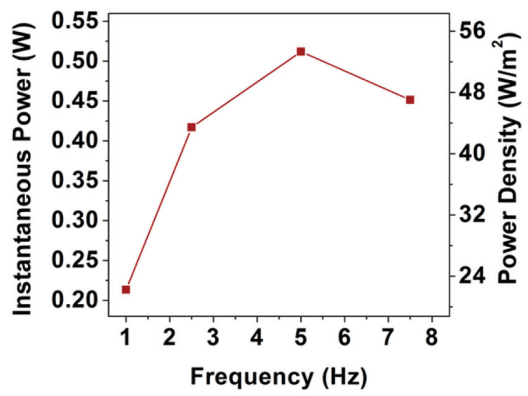

f

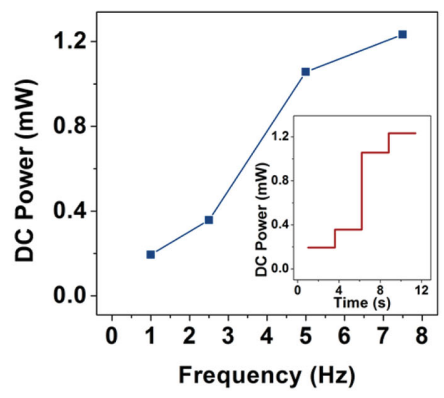

h

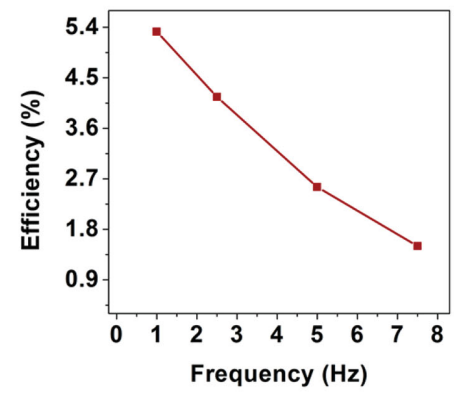

k

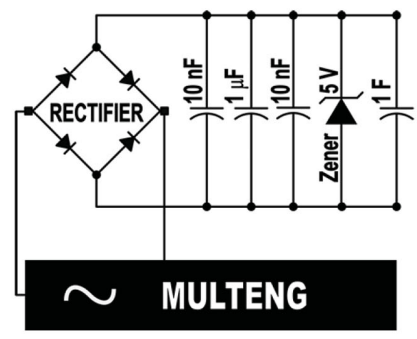

Figure 2. Open circuit characteristics and real-time performance of the MULTENG based on the tapping frequency. a) Frequency-dependent open circuit voltage and b) short circuit current measurements. c) Frequency-dependent instantaneous power and power-density measurements. Rectified d) open circuit voltage and e) short circuit current measurements. f) Frequency-dependent DC power analysis. Inset shows the time-dependent behavior. g) Detailed plot of single OCV peaks in different frequencies which shows obtained outputs with respect to contact and separation sequence of different units of the MULTENG. Orange, blue, and green arrows correspond to motion in unit 1, unit 2, and unit 3, respectively. h) Frequencydependent efficiency that was obtained from open and short circuit measurements. i) Captured image of 38 flashing LEDs powered by the MULTENG. j) Image of 38 enlightened LEDs by the stored energy in the harvesting circuit. k) Schematic representation of the energy harvesting circuit.

means the charging characteristics of different surface scopes can be much different due to inhomogeneous distribution of the nanostructures. Therefore, the layers gain an inhomogeneous charge distribution as a natural effect in each tapping. In addition, each tapping on the MULTENG may move positions of the $\mathrm{As}_{2} \mathrm{Se}_{3}$ nanostructures. This can also slightly affect the charge distribution, especially in high-frequency experiments.
Besides, Al tape electrodes become sticky at higher tapping frequencies because some part of the mechanical motion was converted to heat, and thus Al tape electrodes were avoided for a proper separation of layers. Therefore, the highest instantaneous power and power density were obtained at $5 \mathrm{~Hz}$ as $0.51 \mathrm{~W}$ and $53.4 \mathrm{~W} \mathrm{~m}^{-2}$, respectively (Figure $2 \mathrm{c}$ ). In addition, the sticky part of the tape dramatically decreases the conductivity 
of the electrode and this influences the SCC more than the OCV value. Indeed, we obtained a higher conversion efficiency at a lower frequency. The MULTENG design is more suitable to operate at lower frequencies. DC power is highly used in electronics because billions of low-power-requiring devices are charged using DC power, such as cell phones, music players, portable devices, etc. In power electronics, DC power is the constantly supplied power without any opposite sign polarity (fixed polarity). The output of a MULTENG is similar to alternative current (AC) power with two polarities (negative and positive output) and at a certain frequency, which can be converted in to DC power using transformers, rectifier bridges, and stabilizer capacitors. To calculate the DC power capacity of the MULTENG, we first calculated the rectified current and voltage as shown in Figure 2d,e and we characterized the change in the DC power with respect to frequency. Figure $2 \mathrm{f}$ represents the DC power that was obtained from the MULTENG as a function of tapping frequency. A maximum DC power of $1.23 \mathrm{~mW}$ can be supplied via our MULTENG, when a constant actuation was applied at a frequency of $7.5 \mathrm{~Hz}$. Non-uniform appearance was observed in many output peaks. One of the reasons was that three units of the MULTENG do not touch and separate simultaneously and that causes overlap of multiple output peaks and thus a nonuniform appearance. Contact and separation sequence of the MULTENG units were observed in wider plots of the OCV outputs in Figure $2 \mathrm{~g}$. The color-coded arrows correspond to measured peaks from the motion of different units of the MULTENG. Electrical characterization results, HFR video results, and simulation were perfectly matched. We show detailed OCV and SCC single-peak plots in Figure S12a-c, Supporting Information, which clearly show a contact and separation period. As shown in Figure 1b, there are two terminals set by connecting the three electrodes for the $\mathrm{As}_{2} \mathrm{Se}_{3}$ layer and the other three electrodes for the nanopillar layers. Therefore, generated charges from all the layers pass through the external circuit, which was also connected to all the electrodes in all the units of the MULTENG. This configuration of electrodes affects the device performance in terms of output peak width and height. The DC power, which shows the practicability of the MULTENG in real applications, was increased because sequential contact and separation of multilayers increases the width of peaks. The heights of the peaks corresponding to different units of the MULTENG were not homogeneous. Apparently, the heights of the peaks were related to contact and separation sequences. The maximum peak was always observed from unit 1 in the contact and separation period. This is because induced charges on electrodes of unit 1 were distributed to the external circuit and the electrodes of units 2 and 3. That decreased the charge-induction performance of units 2 and 3, which came into contact before full discharge of unit 1. Obtaining high output peaks from all the units of the MULTENG with the same peak height could be possible by separately and instantaneously discharging all the units of the MULTENG. ${ }^{[32]}$ However, the MULTENG has many conflicts with such a discharging geometry. Considering the motor power as an input energy, the highest energy-conversion efficiency was obtained at a $1 \mathrm{~Hz}$ tapping frequency with $5.3 \%$, and the efficiency was decreased with increasing tapping frequencies (Figure $2 \mathrm{~h}$ ). The MULTENG can directly power up 38 LEDs connected in parallel (Figure $2 \mathrm{i}$ and Video 4 in the Supporting Information). Longer-time-scale SCC plots are given in Figure S13a in the Supporting Information, to show the stability of our device. The MULTENG was connected to a custom-built energy-harvesting circuit to facilitate it as a DC power source and a higher intensity was observed from the same LEDs (Figure 2j) using the energy-harvesting circuit. Instantaneously powered LEDs were observed with lower intensities when compared to the intensities of LEDs powered via the energy-harvesting circuit. As long as the energy-harvesting circuit supplies a DC power to LEDs, it was expected that the LEDs light constantly without any blinking. The energy-harvesting circuit is shown in Figure $2 \mathrm{k}$.

The mechanism of the charge generation in the MULTENG can be explained as follows: once the surfaces of two layers come into contact, surface charge accumulation occurs. This leads to current flow between the two electrodes to compensate for the extra charges injected into them. At the full contact position, the negative charges on the surface of $\mathrm{F}-\mathrm{As}_{2} \mathrm{Se}_{3}$ core-shell nanostructures were fully compensated by the positive counterparts on the surface of PEI nanopillars. Here, the surface roughness on the two surfaces plays a major role in maximizing the triboelectric charge generation. Once the separation of the two surfaces takes place, charge redistribution occurs as a result of charge imbalance inside the layers, this leads to electric current flow between the two terminals of the device opposite in polarity. To develop a theoretical model and describe the electrical characteristics of the device, we assume that the tribocharges were homogeneously distributed on the two surfaces with negligible decay. This assumption is based on the fact that the very short time of contact/separation period during the operation of the device is smaller than typical charge decay time for dielectrics. Detailed information about theoretical model is given in the Supporting Information (see Figure S14). Therefore, the induced potential difference (V) generated between two layers is:

$V=E d$

We developed a finite element simulation model to visualize the change in the induced potential difference in the MULTENG structure (Figure 3a-h). In the simulation, each layer of PEI and $\mathrm{F}-\mathrm{As}_{2} \mathrm{Se}_{3}$ was defined as thin films and surface potential values were chosen according to their position in the triboelectric series. Initially, the outer layers were in contact with each other and they neutralized each other (Figure 3b-e). The middle layers came in contact when the layers were pushed further (Figure $3 \mathrm{f}-\mathrm{h}$ ), as is illustrated in Figure 1b. Finally, all the layers were neutralized as they came in the full contact position. Releasing the layers started the same cycle. The potential difference for a two-layer TENG can be calculated using the following equation:

$V=E_{\mathrm{FAS}} d_{\mathrm{FAS}}+E_{\mathrm{PEI}} d_{\mathrm{PEI}}+E_{\mathrm{air}} d_{\mathrm{air}}$

where $E_{\mathrm{FAS}}, E_{\mathrm{PEI}}$, and $E_{\text {air }}$ are the electric field induced in $\mathrm{F}-\mathrm{As}_{2} \mathrm{Se}_{3}, \mathrm{PEI}$, and air, respectively. $d_{\mathrm{FAS}}, d_{\mathrm{PEI}}$, and $d_{\text {air }}$ are the thicknesses of the respective layers. Recently developed theoretical models assume that the amount of charge (Q) with opposite signs on the electrodes and the generated potential with 

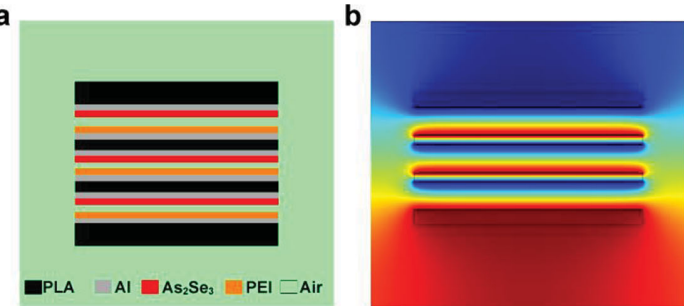

C
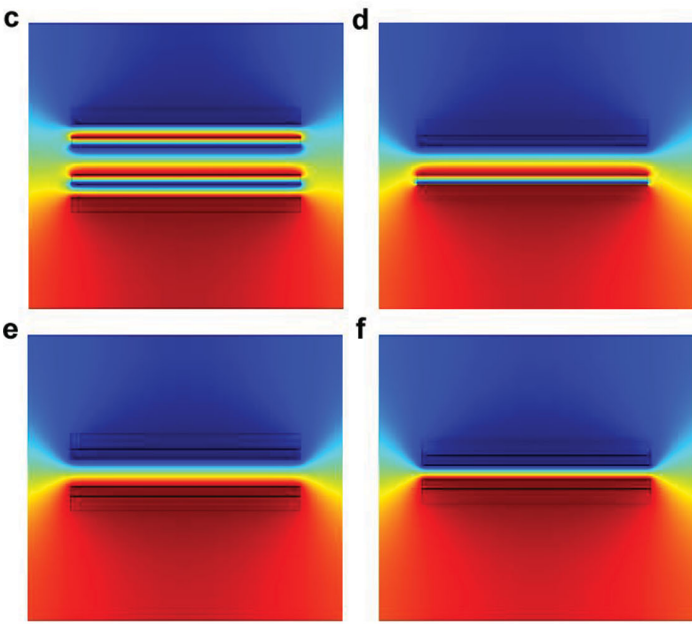

g

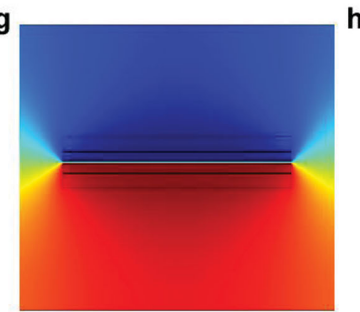

Surface Potential (V)

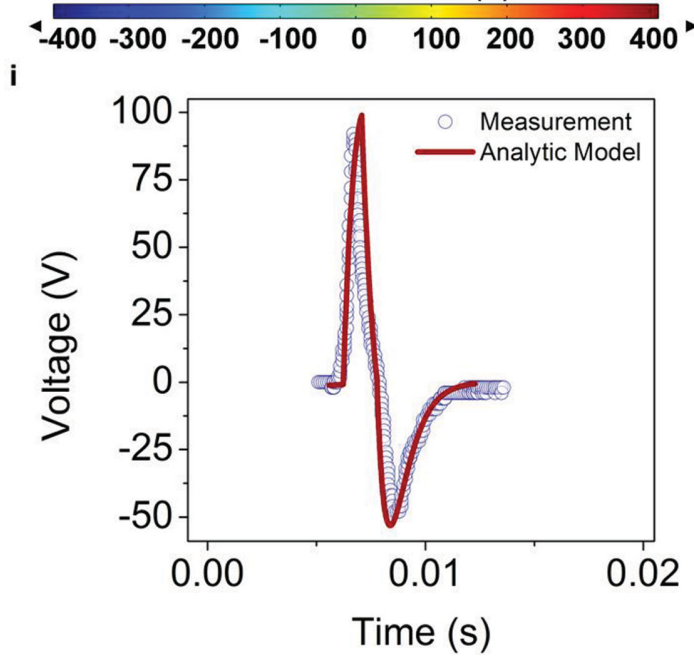

Figure 3. Finite element simulation results to understand the change in the induced potential in accordance with the motion of the layers. a) Representation of the simulation setup. b-e) Initially, the outer layers come in contact (first unit 1 and then unit 3) and the surface potential of the outer layers are balanced. $f-h$ ) All the layers were neutralized when the MULTENG was in full contact by applying further pressure to make the inner layers come in contact as well. i) Comparison of between-measurement from a single layer generator and our analytical model which simulates the open circuit voltage generation. relative displacement $(z(t))$ of the surfaces are related. ${ }^{[33]}$ Considering our device design, the potential difference will only evolve in the direction perpendicular to the surfaces as follows:

$$
V=-\frac{Q d_{\mathrm{FAS}}}{A \varepsilon_{0} \varepsilon_{\mathrm{FAS}}}-\frac{Q d_{\mathrm{PEI}}}{A \varepsilon_{0} \varepsilon_{\mathrm{PEI}}}-\frac{Q z(t)}{A \varepsilon_{0}}+\frac{\sigma z(t)}{\varepsilon_{0}}
$$

where $\varepsilon_{\mathrm{FAS}}$ and $\varepsilon_{\mathrm{PEI}}$ are the relative permittivity of $\mathrm{F}-\mathrm{As}_{2} \mathrm{Se}_{3}$ and PEI, respectively, $\varepsilon_{0}$ is the electrical permittivity of vacuum, $A$ is the surface area of the device, and $\sigma$ is the charge density. We should note that charge transfer is considered zero $(Q=0)$ in the case of open-circuit voltage. The time derivative of the transferred charge gives the short-circuit current when the voltage is considered zero $(V=0)$. We modeled the open-circuit-voltage peak of two-layer generator fabricated using $\mathrm{F}-\mathrm{As}_{2} \mathrm{Se}_{3}$ nanostructures and PEI nanopillars and compared our model with experimental results. The model and the experiment perfectly matched, as seen in Figure 3i. The list of variables used in the model is given in Table S2 in the Supporting Information, and the equations are elaborated in the Supporting Information.

Voltage and current responses of the MULTENG in a moving car (2010 Model Ford Fiesta) were characterized during transportation in Bilkent University Campus with various speeds $\left(15,30,40 \mathrm{~km} \mathrm{~h}^{-1}\right)$. The MULTENG was positioned on the front panel of the car. An Al block $1.3 \mathrm{~kg}$ in weight, $60 \mathrm{~mm}$ in height, $25 \mathrm{~mm}$ in width, and $280 \mathrm{~mm}$ in length was located perpendicularly on top of the MULTENG as the centers of the device and the metal block coincide (Figure 4a). Because the Al block pushes on top of the device, the initial position of the MULTENG was set as full contact with no response and reaction, as shown in the fourth stage of the triboelectric cycle in Figure 1b. As the car moved forward at various speeds, the Al block on the top tilted the layers of the MULTENG backward and forward which caused partially separation and contact of layers. Therefore, small jumps cause quick and weak separations between the layers while the car moves in a routine way. So, the input mechanical energy here can be low and the output signal can be very complex because the response of the MULTENG to car vibration depends on many parameters, such as the roughness of the road, the car model, the skills of driver, and the geometry of the MULTENG. The highest instantaneous power and power density were obtained at a $40 \mathrm{~km} \mathrm{~h}^{-1}$ speed as $127 \mu \mathrm{W}$ and $13.23 \mathrm{~mW} \mathrm{~m}^{-2}$, respectively, as seen in Figure $4 \mathrm{~b}$. The instantaneous power and power density were increased with increasing car speed. The OCV and SCC values were measured as the car passed over speed bumps (maximum $13 \mathrm{~cm}$ in height and $150 \mathrm{~cm}$ in width) at 15,30 , and $40 \mathrm{~km} \mathrm{~h}^{-1}$ speeds. The driver of the car slightly pushed the breaks just before passing through the speed bumps. This action caused the MULTENG to tilt backward and separate its layers. As the car moved further on the speed bumps, the vibration and separation also increased due to the greater inclination. The MULTENG was tilted forward while the car leaped over the top of the speed bump to access the flat asphalt. OCV and SCC graphs with respect to car speed while passing over a speed bump are shown in Figure 4c-h. The maximum peak-to-peak OCV was measured as $61.96 \mathrm{~V}$ at $40 \mathrm{~km} \mathrm{~h}^{-1}$ speed as shown in Figure $4 \mathrm{~g}$. The maximum peakto-peak SCC was measured as $2.05 \mu \mathrm{A}$ at $40 \mathrm{~km} \mathrm{~h}^{-1}$ speed, as shown in Figure $4 \mathrm{~h}$. Since the response of the MULTENG to 

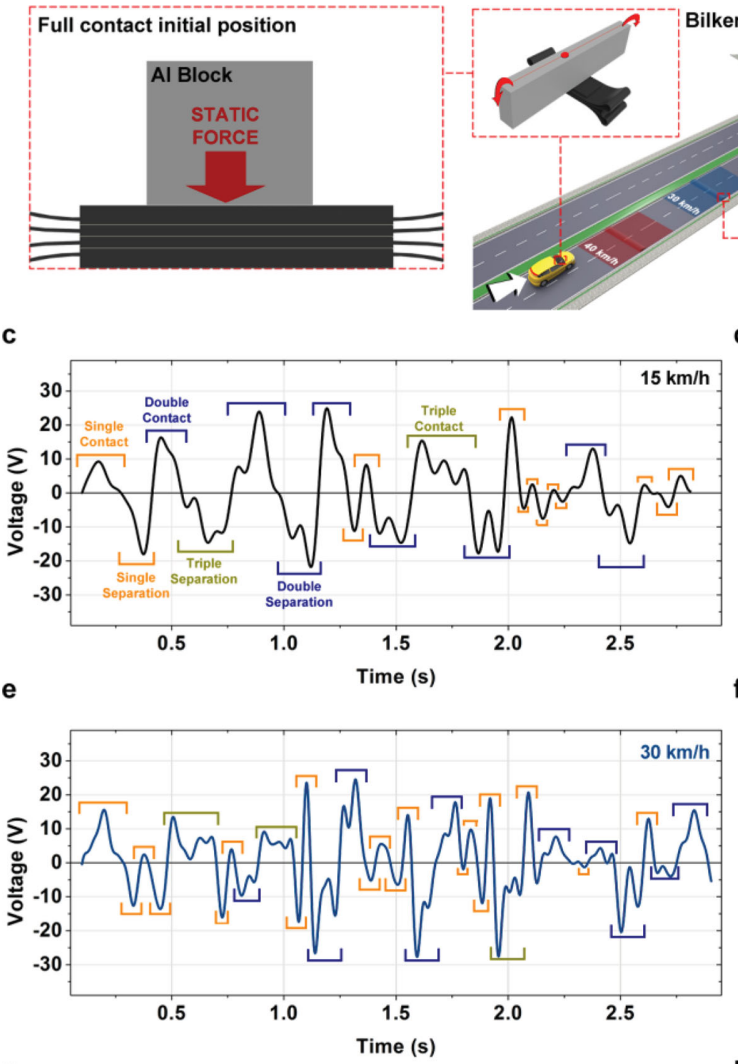

g

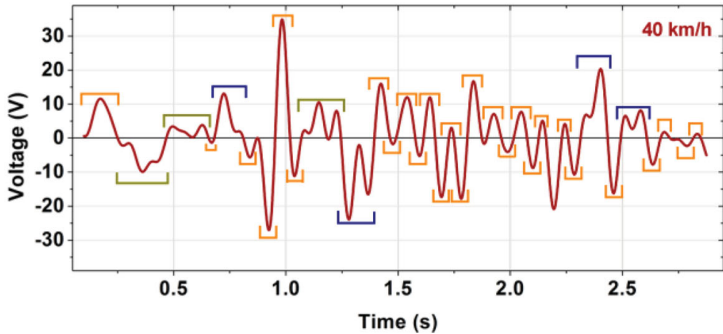

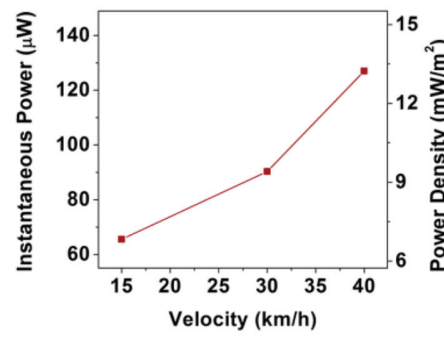

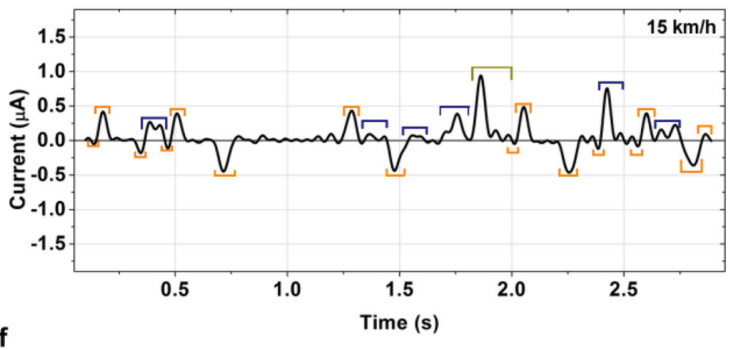

h
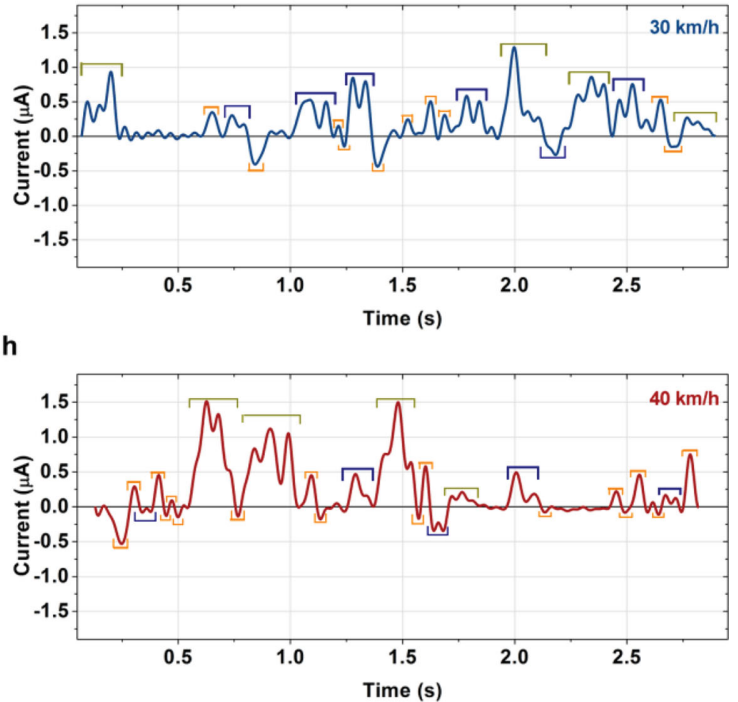

Figure 4. Electrical characterizations of the MULTENG, activated from the motion of a moving car. a) Schematic representation of driving route in the university campus. Inset shows the squeezed MULTENG in full contact position. Inset shows the bump dimensions on the route. b) Instantaneous power and power density measurements as a function of car velocity. $c-h$ ) Open-circuit voltage and short-circuit current measurements with respect to car velocity. The orange, blue, and green colors correspond to single, double, and triple contact and separation in different units of the MULTENG, respectively. Detailed motion of the MULTENG is given in Figure S15 and S16 in the Supporting Information.

car vibration was complicated because of the multiple contact and separation sequences, we pointed single, double, and triple contacts and separations in different units of the MULTENG during the measurements. Our observations about the motion of the MULTENG in car measurements are shown in Figure S15 and S16, Supporting Information. We observed two types of motions (linear and angular) in the MULTENG units. Linear motions caused a strong response in the OCV and SCC and angular motions caused weak responses.

Reaction of the MULTENG to sound at different frequencies was also measured. Before the tests, a constant initial stress was applied to the MULTENG from the sides for closing the gaps between the layers to around $1 \mathrm{~mm}$, as seen in Figure 5a. The squeezed MULTENG was located $10 \mathrm{~cm}$ above from the sound source which has an acoustic power $\left(P_{\mathrm{ac}}\right)$ of
$1.9 \mathrm{~W}$ corresponding to a $100 \mathrm{~dB}$ sound power level $\left(L_{\mathrm{W}}\right)$. The acoustic power and sound power level were calculated using the following equations:

$P_{\mathrm{ac}}=I S$

$L_{\mathrm{W}}=10 \log _{10}\left(\frac{P_{\mathrm{ac}}}{P_{0}}\right)$

where $I$ is the sound intensity, $S$ is the area of the source, and $P_{0}$ is the reference sound power in air as the threshold of hearing $\left(10^{-12} \mathrm{~W}\right)$. The acoustic power and sound power level are the characteristics of the sound source. In order to understand the effect of sound pressure on the MULTENG, 

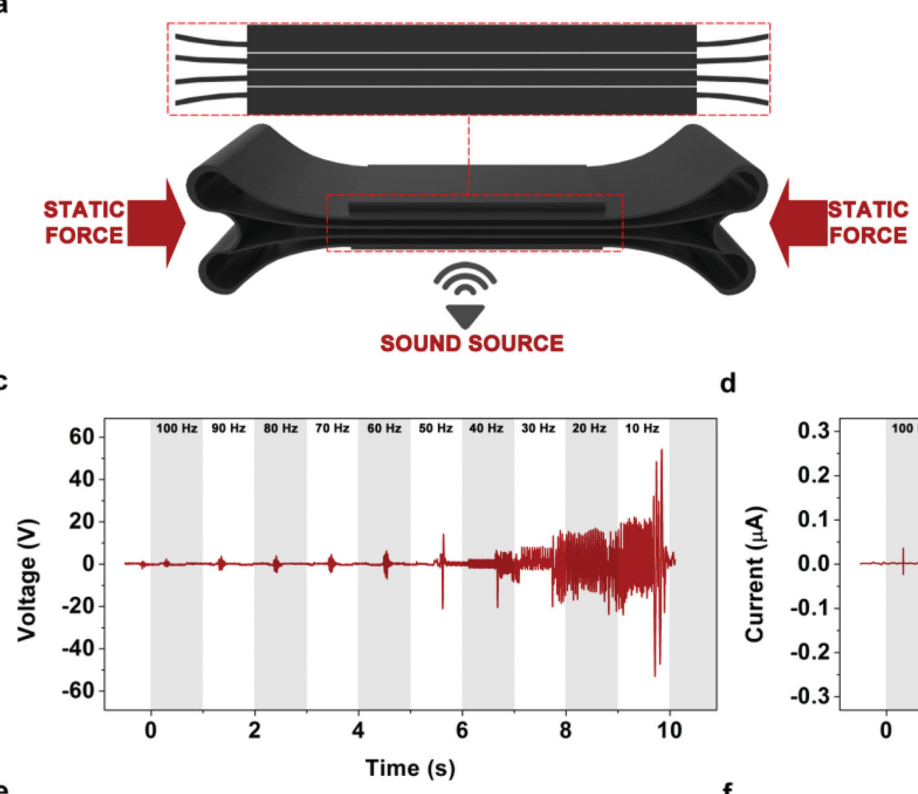

b

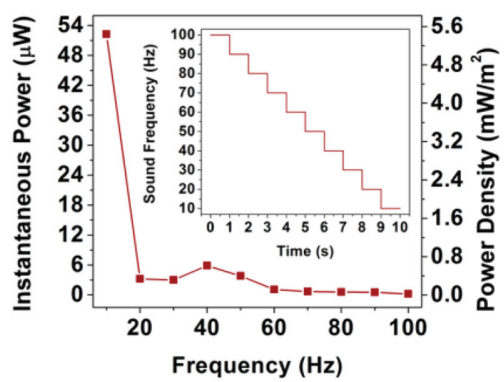

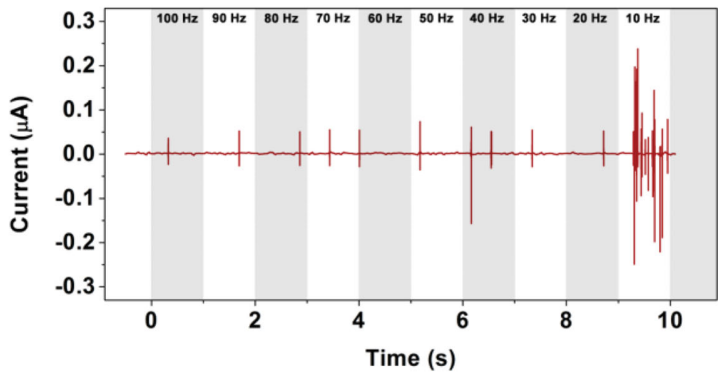
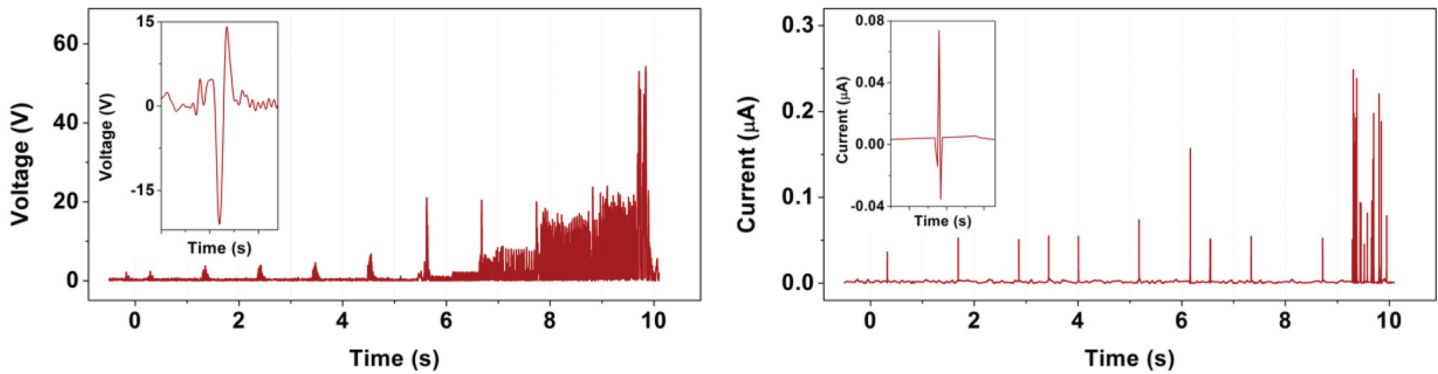

Figure 5. Electrical characterization of acoustically actuated MULTENG. a) Schematic representation of sound actuation. b) Sound-frequency-dependent instantaneous power and power density measurements. Inset: Change input sound frequency (Hz) over time. Sound-frequency-dependent c) open circuit voltage and d) short circuit current measurements. e) Rectified open circuit voltage and single peak open circuit voltage. f) Rectified short circuit current and single peak short circuit current.

we calculated sound pressure level $\left(L_{\mathrm{p}}\right)$ and sound pressure $(p)$ which is related to distance between source and the MULTENG $(r)$ and sound propagation characteristics $(q)$ using the following equations:

$L_{\mathrm{P}}=L_{\mathrm{W}}-10\left|\log _{10}\left(\frac{q}{4 \pi r^{2}}\right)\right|$

$p=\sqrt{\frac{q \rho c P_{\mathrm{ac}}}{4 \pi r^{2}}}$

where $\rho$ is the density of air $\left(1.2041 \mathrm{~kg} \mathrm{~m}^{-3}\right)$ at room temperature, and $c$ is the speed of sound in air $\left(343.22 \mathrm{~m} \mathrm{~s}^{-1}\right)$ at room temperature. In our case, $r=0.1 \mathrm{~m}$ and the propagation characteristics are half sphere $(q=2)$. Using the given formula and parameters, we calculated the sound pressure level as $87.98 \mathrm{~dB}$ and the sound pressure as $111.8 \mathrm{~N} \mathrm{~m}^{-2}$, which causes separation and contact between the layers of the MULTENG. OCV and SCC values were measured and the instantaneous power output of the MULTENG was calculated as the sound frequency decreased from 100 to $10 \mathrm{~Hz}$ with $10 \mathrm{~Hz}$ steps and
$1 \mathrm{~s}$ duration as shown in Figure 5b. The highest instantaneous power and power density were also obtained at $10 \mathrm{~Hz}$ as $52.5 \mu \mathrm{W}$ and $5.47 \mathrm{~mW} \mathrm{~m}^{-2}$, respectively (Figure $5 \mathrm{~b}$ ). Peaks with an increasing fashion were obtained with decreasing sound frequency at each $1 \mathrm{~s}$ frame. A significant increase in the voltage signal was observed when the sound frequency was reduced to $50 \mathrm{~Hz}$, which proves that our MULTENG design is more suitable to operating at lower frequencies. Indeed, the OCV value reached the maximum peak-to-peak $107.3 \mathrm{~V}$ at $10 \mathrm{~Hz}$ (Figure 5c). Validating these findings, there was no significant SCC signal that occurred beyond $10 \mathrm{~Hz}$ sound frequency. The maximum peak-to-peak SCC value was measured as $0.49 \mu \mathrm{A}$ at $10 \mathrm{~Hz}$ (Figure 5d). Rectified OCV and SCC peaks are given in Figure 5e,f. Besides, single-peak OCV and SCC are given in Figure $5 \mathrm{e}$, f insets for a better view of peaks.

In conclusion, we have developed a multilayered triboelectric generator based on $\mathrm{As}_{2} \mathrm{Se}_{3}$ core-shell nanostructures and PEI nanopillars, which were produced by iterative size reduction and melt infiltration techniques, respectively. Triboelectric response of a new materials set was characterized and we deduced that increasing the fluorine content in the materials increases the triboelectric response. The MULTENG was 
activated using mechanical tapping, car vibration, and sound with different frequencies and forces. The mechanical tapping force was the most powerful input among these three conditions and we obtained the highest signals in the mechanical tapping condition, since it made a stronger contact between layers. The results are summarized in Table S3 in the Supporting Information. The maximum peak-to-peak OCV and SCC were obtained as $396 \mathrm{~V}$ at $7.5 \mathrm{~Hz}$ and $1.62 \mathrm{~mA}$ at $2.5 \mathrm{~Hz}$. The energy conversion efficiency of the device was calculated as $5.3 \%$ at $1 \mathrm{~Hz}$ tapping frequency and it was decreased with increasing tapping frequency. The MULTENG can supply $1.23 \mathrm{~mW}$ constant DC power and directly illuminate 38 LEDs as well as charge a capacitor via an energy-harvesting circuit. The voltage and current signals were recorded in a car during transportation on campus road and leaping through speed bumps at various speeds. The signal intensities and output power density (13.23 $\mathrm{mW} \mathrm{m}^{-2}$ at $40 \mathrm{~km} \mathrm{~h}^{-1}$ speed) were increased with increasing car speed. Measurements at sound frequencies between 10 and $100 \mathrm{~Hz}$ revealed that our device has a better response at $10 \mathrm{~Hz}$ with peak-to-peak $107.3 \mathrm{~V} \mathrm{OCV}$ and peak-topeak $0.49 \mu \mathrm{A}$ SCC. The output power density (max. $5.47 \mathrm{~mW} \mathrm{~m}^{-2}$ ) of the MULTENG decreased with increasing sound frequency. The new material and processes proposed in this study such as the fluorine-based coating, which enhance the triboelectric properties of materials, will instigate new theoretical studies to explain the underlying mechanism of the triboelectric effect. Demonstration of the MULTENG was aimed for charging portable electronic devices such as cell phones and music players with an energy-harvesting circuit.

\section{Supporting Information}

Supporting Information is available from the Wiley Online Library or from the author.

\section{Acknowledgements}

The authors thank Murat Dere for his help during the preform preparation, fiber drawing, and electronic circuit construction and S. Ali Yasar for constructing the taping system and sound measurement setup. The authors also thank Fatih Büyükserin for the AAO membranes. This work was partially supported by TUBITAK under Project Nos. 111A015 and 111 T696. The research leading to these results has received funding from the European Research Council under the European Union's Seventh Framework Programme (FP/2007-2013)/ERC Grant Agreement No. 307357. M.B. acknowledges partial support from the Turkish Academy of Sciences (TUBA).

Received: December 31, 2014 Revised: January 27, 2015 Published online: February 26, 2015

[1] I. F. Akyildiz, W. Su, Y. Sankarasubramaniam, E. Cayirci, Comput. Networks 2002, 38, 393.

[2] H. Klauk, U. Zschieschang, J. Pflaum, M. Halik, Nature 2007, 445, 745.
[3] T. Someya, T. Sekitani, S. Iba, Y. Kato, H. Kawaguchi, T. Sakurai, Proc. Natl. Acad. Sci. USA 2004, 101, 9966.

[4] T. Sekitani, M. Takamiya, Y. Noguchi, S. Nakano, Y. Kato, T. Sakurai, T. Someya, Nat. Mater. 2007, 6, 413.

[5] M. Kanik, O. Aktas, H. S. Sen, E. Durgun, M. Bayindir, ACS Nano 2014, 8, 9311

[6] L. Persano, C. Dagdeviren, Y. Su, Y. Zhang, S. Girardo, D. Pisignano, Y. Huang, J. A. Rogers, Nat. Commun. 2013, 4, 1633.

[7] L. Gu, N. Cui, L. Cheng, Q. Xu, S. Bai, M. Yuan, W. Wu, J. Liu, Y. Zhao, F. Ma, Y. Qin, Z. L. Wang, Nano Lett. 2013, 13, 91.

[8] C. Chang, V. H. Tran, J. Wang, Y.-K. Fuh, L. Lin, Nano Lett. 2010, 10, 726.

[9] S. Cha, S. M. Kim, H. Kim, J. Ku, J. I. Sohn, Y. J. Park, B. G. Song, M. H. Jung, E. K. Lee, B. L. Choi, J. J. Park, Z. L. Wang, J. M. Kim, K. Kim, Nano Lett. 2011, 11, 5142.

[10] Y. Saito, H. Takao, T. Tani, T. Nonoyama, K. Takatori, T. Homma, T. Nagaya, M. Nakamura, Nature 2004, 432, 84.

[11] M. Yaman, T. Khudiyev, E. Ozgur, M. Kanik, O. Aktas, E. O. Ozgur, H. Deniz, E. Korkut, M. Bayindir, Nat. Mater. 2011, 10, 494.

[12] G. Zhu, Z.-H. Lin, Q. Jing, P. Bai, C. Pan, Y. Yang, Y. Zhou, Z. L. Wang, Nano Lett. 2013, 13, 847 .

[13] G. Zhu, J. Chen, T. Zhang, Q. Jing, Z. L. Wang, Nat. Commun. 2014, $5,3426$.

[14] W. Yang, J. Chen, Q. Jing, J. Yang, X. Wen, Y. Su, G. Zhu, P. Bai, Z. L. Wang, Adv. Funct. Mater. 2014, 24, 4090.

[15] Y. Xie, S. Wang, L. Lin, Q. Jing, Z.-H. Lin, S. Niu, Z. Wu, Z. L. Wang, ACS Nano 2013, 7, 7119.

[16] Z.-H. Lin, Y. Xie, Y. Yang, S. Wang, G. Zhu, Z. L. Wang, ACS Nano 2013, 7, 4554.

[17] W. Du, X. Han, L. Lin, M. Chen, X. Li, C. Pan, Z. L. Wang, Adv. Energy Mater. 2014, 4, 1301592.

[18] G. Cheng, Z.-H. Lin, Z. Du, Z. L. Wang, Adv. Funct. Mater. 2014, 24, 2892.

[19] P. Bai, G. Zhu, Z.-H. Lin, Q. Jing, J. Chen, G. Zhang, J. Ma, Z. L. Wang, ACS Nano 2013, 7, 3713.

[20] Z. L. Wang, ACS Nano 2013, 7, 9533.

[21] J. Yang, J. Chen, Y. Liu, W. Yang, Y. Su, Z. L. Wang, ACS Nano 2014, 8, 2649.

[22] S. Matsusaka, H. Maruyama, T. Matsuyama, M. Ghadiri, Chem. Eng. Sci. 2010, 65, 5781

[23] H. T. Baytekin, A. Z. Patashinski, M. Branicki, B. Baytekin, S. Soh, B. A. Grzybowski, Science 2011, 333, 308.

[24] L. S. McCarty, G. M. Whitesides, Angew. Chem. Int. Ed. 2008, 47, 2188.

[25] H. T. Baytekin, B. Baytekin, T. M. Hermans, B. Kowalczyk, B. A. Grzybowski, Science 2013, 341, 1368.

[26] B. Baytekin, H. T. Baytekin, B. A. Grzybowski, J. Am. Chem. Soc. 2012, 134, 7223.

[27] O. Aktas, E. Ozgur, O. Tobail, M. Kanik, E. Huseyinoglu, M. Bayindir, Adv. Opt. Mater. 2014, 2, 618.

[28] A. F. Abouraddy, M. Bayindir, G. Benoit, S. D. Hart, K. Kuriki, N. Orf, O. Shapira, F. Sorin, B. Temelkuran, Y. Fink, Nat. Mater. 2007, 6, 336

[29] M. Shurgalin, C. Anastassiou, Biomed. Instrum. Technol. 2008, 42, 318.

[30] B. Daglar, T. Khudiyev, G. B. Demirel, F. Buyukserin, M. Bayindir, J. Mater. Chem. C 2013, 1, 7842.

[31] B. Daglar, G. B. Demirel, T. Khudiyev, T. Dogan, O. Tobail, S. Altuntas, F. Buyukserin, M. Bayindir, Nanoscale 2014, 6, 12710.

[32] G. Cheng, Z.-H. Lin, L. Lin, Z.-L. Du, Z. L. Wang, ACS Nano 2013, 7, 7383.

[33] S. Niu, S. Wang, L. Lin, Y. Liu, Y. S. Zhou, Y. Hu, Z. L. Wang, Energy Environ. Sci. 2013, 6, 3576. 